sarcoidosis was more frequently observed in those patients with a previous history of neoplasia, while the association with hematological neoplasms was linked to a higher frequency of sarcoidosis involving ENT and bone marrow.

Disclosure of Interest: None declared

DOI: 10.1136/annrheumdis-2017-eular.3700

\section{FRI0616 EYE MANIFESTATIONS OF PATIENTS WITH MUCKLE-WELLS} SYNDROME

S. Cekic ${ }^{1}$, O. Yalcinbayir ${ }^{2}$, S.S. Kilic ${ }^{1} .{ }^{1}$ Pediatric Rheumatology;

${ }^{2}$ Ophtahlmatology, Uludag University Medical Faculty, Bursa, Turkey

Background: CAPS is a rare autoinflammatory disease associated with mutations in the CIAS1 gene, encoding for NLRP3 that result in overactivation of the inflammasome and systemic inflammation. Muckle-Wells syndrome (MWS) is a rare autosomal dominant disease which causes episodic fever attacks, sensorineural deafness, recurrent hives, arthritis and eye involvement.

Objectives: Here we present the findings of eye involvement in a family whose 11 members have MWS.

Methods: Clinical data was collected during the course of ongoing patient care.

Results: We evaluated the clinical features of 11 patients who were referred to a tertiary care center. The median age of the patients was 25 years (range: 9-65). The ratio of females /males was 1.2 (6/5). All patients had arthritis with exacerbation on exposure to cold and recurrent episodes of pink eye. The median age of onset of ocular involvement was 8 years (2-45). We observed severe eye involvement in $36 \%$ of our cases, including band keratopathy, severe damage of corneal stroma and neovascularization. Corneal involvement and clouding was detected in four patient. Two of those had the diagnosis of keratoconus as well. Patients with keratoconus had corneal scarring due to corneal hydrops verified with corneal topography. The other two patients with corneal clouding had bant keratopathy. One of those patient was a 17 year old girl who had recurrent uveitis with hypopyon which necessiated the use of intravitreal dexamethasone implant. She also had posterior synechia of the iris to the lens. The other eye of that patient had signs of phthisis bulbi. The other patient with bant keraopathy was 46 years old male who had optic atrophy and tractional fibrovascular membranes at the posterior pole of the eye. Anakinra was used for treatment of 5 cases, and canakinumab of 3 cases. It was observed that the frequency of conjunctivitis decreased after anti IL-1 therapy. There was no mutation detected in the study of MEFV (all exons), TNFRSF1A (exons 2 to 7), MVK (all exons), NLRP3 (all exons), NOD2 (exons 4, 8 and 9) and PSTPIP1 (exons 10 and 11) genes.

Conclusions: In this study, it has been shown that eye findings related to MWS can vary from conjunctivitis to severe uveitis. We want to emphasize that ocular involvement in MWS should be carefully assessed, since it can lead to visual impairment.

Disclosure of Interest: None declared

DOI: 10.1136/annrheumdis-2017-eular.3352

\section{FRI0617 DIAGNOSTIC SENSITIVITY OF CUTOFF VALUES OF IGG4-POSITIVE PLASMA CELL NUMBER AND IGG4-POSITIVE/CD138-POSITIVE CELL RATIO IN TYPICAL MULTIPLE LESIONS OF PATIENTS WITH IGG4-RELATED DISEASE}

S. Tsuge, I. Mizushima, Y. Fujisawa, S. Hara, F. Suzuki, K. Ito, H. Fujii, K. Yamada, M. Kawano. Division of Rheumatology, Department of Cardiovascular and Internal Medicine, Kanazawa University hospital, Kanazawa, Kanazawa, Japan

Background: Immunoglobulin G4-related disease (IgG4-RD) is a recently recognized systemic inflammatory disease with multi-organ involvement [1]. Diagnostically, two of the most important hallmarks of IgG4-RD are high IgG4-positive plasma cell (PC) counts and high IgG4/lgG ratios in affected organs. Although the International consensus statement (ICS) on the pathology of IgG4-RD adopted different IgG4-positive PC counts among affected organs for the diagnosis to differentiate IgG4-RD mimickers from IgG4-RD [2], histological and immunohistochemical findings of the specimens from not only one but multiple organs in the same patient has not been evaluated.

Objectives: This study aimed to investigate the diagnostic sensitivity of the cutoff values of IgG4-positive PC number and lgG4-positive/CD138-positive cell ratio proposed by the International consensus statement (ICS) on the pathology of IgG4-RD in typical multiple lesions of patients with IgG4-RD.

Methods: We evaluated IgG4-positive PC number and IgG4-positive/CD138positive cell ratio in 35 samples from 16 IgG4-RD patients having more than two typical lesions of IgG4-RD.

Results: We evaluated twelve submandibular, eleven ophthalmic, four skin, four kidney, two pancreatic, and one bronchus and prostate lesion each in 16 patients with typical clinical, serological, and radiographic features. Concerning IgG4+ PC number per high power field, most ophthalmic (8/11), kidney (4/4), pancreatic $(2 / 2)$, and bronchial lesions (1/1) fulfilled the cutoff value of ICS, whereas many of the submandibular (5/12) and skin lesions (0/4) did not. In contrast to the absolute number, almost all lesions fulfilled the cutoff value of IgG4+/CD138+ cell ratio. In five cases, only one or two lesions in the same patient fulfilled the cutoff value of ICS, while the others did not.
Conclusions: These results suggest that ICS criteria have different sensitivities among the affected organs in diagnosing IgG4-RD.

References:

[1] Stone JH, et al. IgG4-related disease. N Engl J Med. 2012;366:539-51.

[2] Deshpande V, et al. Consensus statement on the pathology of IgG4-related disease. Mod Pathol. 2012;25:1181-92.

Disclosure of Interest: None declared

DOI: 10.1136/annrheumdis-2017-eular.4170

\section{FRI0618 ADALIMUMAB IN NON-INFECTIOUS UVEITIS - EFFICACY ACROSS DIFFERENT ETIOLOGIES IN THE VISUAL I AND VISUAL II TRIALS}

P.T. Merrill ${ }^{1}$, A. Vitale ${ }^{2}$, M. Zierhut ${ }^{3}$, E. Forton ${ }^{4}$, H. Goto ${ }^{5}$, M. Kron ${ }^{6}$, S. Tari ${ }^{7}$, S. Pathai ${ }^{8} \cdot{ }^{1}$ Rush University Medical Center, Chicago; ${ }^{2}$ University of Utah, Salt Lake City, United States: ${ }^{3}$ University of Tuebingen, Center of Ophthalmology, Tuebingen, Germany; ${ }^{4}$ University of Montreal, Montreal, Canada; ${ }^{5}$ Tokyo Medical University, Tokyo, Japan; ${ }^{6}$ AbbVie Deutschland GmbH \& Co KG, Ludwigshafen, Germany; ${ }^{7}$ AbbVie Inc., North Chicago, United States; ${ }^{8}$ AbbVie Ltd, Maidenhead, United Kingdom

Background: There is increasing interest in understanding the efficacy of adalimumab across different etiologies of uveitis. No prospective analysis has been conducted to date to determine the efficacy of adalimumab among non-infectious uveitis patients with different etiologies.

Objectives: To assess adalimumab (ADA) efficacy in active and inactive, noninfectious uveitis across different etiologies in patients who were recruited as part of the VISUAL program.

Methods: Exploratory data analyses from two global phase 3, double-masked trials: VISUAL I (patients with active uveitis despite $>2$ weeks of prednisone 10-60 mg/day) and VISUAL II (patients with inactive disease dependent on 10-35 $\mathrm{mg} /$ day of prednisone to maintain inactivity) were performed. Patients received placebo (PBO) or ADA subcutaneously ( $80 \mathrm{mg}$ week 0 , followed by $40 \mathrm{mg}$ every other week from week 1 up to 80 weeks). In VISUAL I, all patients received a prednisone burst followed by taper to $0 \mathrm{mg}$ by week 15 . In VISUAL II, prednisone taper to $0 \mathrm{mg}$ was mandatory by week 19 . The primary endpoint was time to treatment failure (TF) at or after week 6 for VISUAL I; and at or after week 2 for VISUAL II ${ }^{1,2}$. For this analysis, patients were categorized into different uveitis etiologies which they presented at study entry. Hazard ratios (HR) for time to TF were obtained for each uveitis etiology.

Results: The efficacy of ADA was significantly greater than PBO in the largest subgroup of patients with Idiopathic/other uveitis (VISUAL I: 103 and VISUAL II: 90) etiology in both VISUAL I' and VISUAL II trials. All other subgroups showed

Figure: Hazard ratios of time to treatment failure by uveitis etiologies. VISUALI (A) and VISUAL I* (B) clinical trials.

A. VISUALI

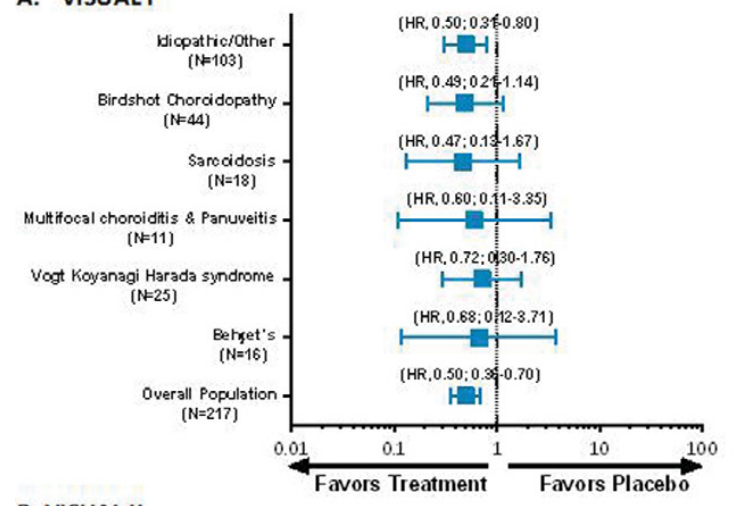

B. VISUAL II

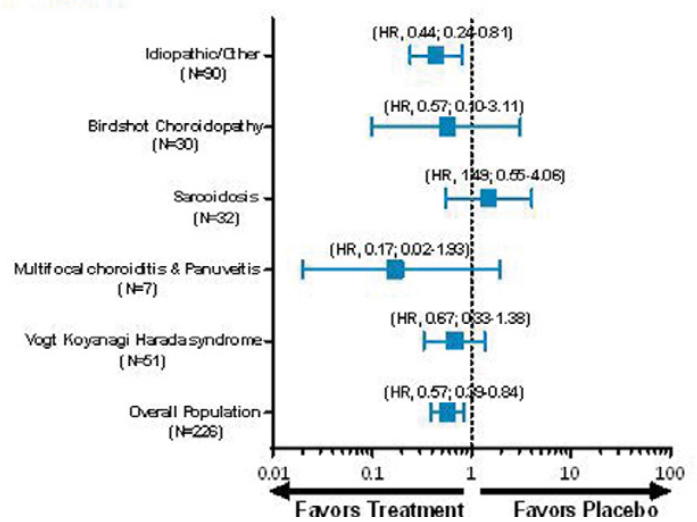


a trend in favor of ADA, except for Sarcoidosis subgroup in the VISUAL II trial (Figure). Overall safety for both trials has been previously reported ${ }^{1,2}$.

Conclusions: These exploratory analyses from the VISUAL I and VISUAL II trials show significantly higher efficacy in ADA-treated patients over PBO in "idiopathic/other" diagnoses of patients with both active and inactive non-infectious uveitis. Furthermore, across different uveitis etiologies, these analyses suggest that ADA-treated patients had a prolonged time to treatment failure compared to PBO.

References:

[1] Jaffe GJ, Dick AD, Brezin AP, et al. N Engl J Med (2016); 375:932-43.

[2] Nguyen QD, Merrill PT, Jaffe GJ, et al. The Lancet (2016); 388(10050): 1183-92.

Acknowledgements: AbbVie funded the VISUAL I and VISUAL II studies and provided writing support. All authors contributed to the development of the content. The authors and AbbVie reviewed and approved the abstract. The authors maintained control over the final content. Medical writing assistance was provided by Gaurav Patki, PhD of AbbVie Inc.

Disclosure of Interest: P. T. Merrill Consultant for: Santen, AbbVie, A. Vitale Consultant for: ACIONT, M. Zierhut Consultant for: AbbVie and Santen, E. Forton Consultant for: AbbVie, Alcon and Allergan, H. Goto Consultant for: AbbVie, M. Kron Shareholder of: AbbVie, Employee of: AbbVie, S. Tari Shareholder of: AbbVie, Employee of: AbbVie, S. Pathai Shareholder of: AbbVie, Employee of: AbbVie

DOI: 10.1136/annrheumdis-2017-eular.2389

\section{FRI0619 COMPARING CANAKIMUMAB AND ANAKINRA IN YOUNG} GREEK WOMEN WITH RESISTANT RECURRING PERICARDITIS

P. Kiryttopoulos ${ }^{1}$, T. Michailidis ${ }^{1}$, E. Papchianou ${ }^{1}$, M. Charalampidis ${ }^{2}$, P. Dimitriadis ${ }^{3}$, A. Agorastos ${ }^{4}$, T. Mpektsis ${ }^{1} .{ }^{1}$ Department of Internal Medicine, General Hospital of Veria, Veria; ${ }^{2}$ Gastroenterology Department, Theageneio Hospital of Thessaloniki, Thessaloniki; ${ }^{3}$ Health Center of Alexandria, Alexandria, Greece; ${ }^{4}$ Oncology Department, St. Thomas Hospital, London, United Kingdom

Background: Resistant - recurring pericarditis (RRP) is often associated with autoinflammatory syndromes. Conventional therapy with glucocorticoids and colchicine is often enough unsuccessful, thus nowadays the use of anakinra in such cases is becoming common every - day practice.

Objectives: Our goal was to determine whether RRP was associated with gene mutations relevant with autoinflammatory diseases and then compare the effectiveness of canakimumab and anakinra.

Methods: We studied 18 patients with RRP (all women, from 14 to 36 years old) determing the existence of mutation from the infevers database ( 25 genes, from MEFV and TNFRSF1A to MVK and SLC 29A3, all related with autoinflammatory diseases). All the patients were proven positive to one of the above mutations (all heterozygous) and then treatment with anakinra ( $n=9,100 \mathrm{mg} \mathrm{sc}$ once daily) and canakimumab ( $n=9,150 \mathrm{mg}$ sc $q 4 w k$ ) was administered.

Results: During the study (3 years), all anakinra patients stopped receiving per os glucocorticoids and were free of any new pericarditis episodes. 3 of them $(33 \%)$ stopped any treatment after a year of anakinra therapy and none was presented with any skin reactions.

On the contrary, all canakimumab patients were not able to free themselves of the glucocorticoid treatment, since at least one new episode of pericarditis to each patient was recorded. Two of them were free of pericarditis the last year of the study (though being treated with $2,5 \mathrm{mg}$ prednisolone per os daily).

Statistically speaking, the use of IL-1R antagonist in recurrent - resistant pericarditis associated with autoinflammatory mutations was proven much more successful $(p<0,01)$ than the use of the IL-1b Mab.

Conclusions: Though the sample of patients $(n=18)$ was too small in order to set us able to reach any safe conclusions, it is quite possible that anakinra may be one effective solution that can prevent the long term administration of corticoids to patients with recurrent pericarditis Canakimumab was not recorded with similar positive results.

Disclosure of Interest: None declared

DOI: 10.1136/annrheumdis-2017-eular.6289

\section{FRI0620 MIR-204-3P INHIBITS THE PRODUCTION OF TLR4-RELATED CYTOKINES IN FAMILIAL MEDITERRANEAN FEVER BY TARGETING THE PIK3 SIGNALING PATHWAY}

T. Koga ${ }^{1,2}$, K. Migita $^{3}$, M. Umeda ${ }^{1}$, F. Nonaka ${ }^{4}$, S.-Y. Kawashiri ${ }^{1}$, N. Iwamoto ${ }^{1}$ K. Ichinose ${ }^{1}$, M. Tamai ${ }^{1}$, H. Nakamura ${ }^{1}$, T. Origuchi ${ }^{1}$, K. Eguchi ${ }^{5}$,

A. Kawakami ${ }^{1}$. ${ }^{1}$ Department of Immunology and Rheumatology, Unit of

Advanced Preventive Medical Sciences, Nagasaki University Graduate School of

Biomedical Sciences; ${ }^{2}$ Center for Bioinformatics and Molecular Medicine, Nagasaki University Graduate School of Biomedical Sciences, Nagasaki;

${ }^{3}$ Fukushima Medical University School of Medicine, Fukushima Medical University School of Medicine; ${ }^{4}$ Department of Internal Medicine, Sasebo City General Hospital; ${ }^{5}$ Center for Rheumatic Disease, Sasebo Chuo Hospital, Sasebo, Japan

Background: MicroRNAs (miRNAs) are endogenous small RNAs and posttranscriptionally regulate gene expression by pairing with target. There has been emerging evidence showing the association of aberrantly expressed circulating miRNAs in the serum with the pathogenesis or progression of diseases including cancer and autoimmune disease. Although a number of circulating miRNAs associated with inflammation have been identified, the roles of them in patients with FMF and the underlying mechanism remain to be elucidated.

Objectives: The aim of this study was to identify a serum miRNAs profile and potential biomarkers in FMF and clarify their gene targets for understanding the pathogenesis of autoinflammatory diseases.

Methods: We performed miRNA microarray in the serum from FMF in attack and in remission. We subsequently examined the expression of miRNAs that varied before and after the attack in macrophages derived from THP-1 cells stimulated with toll-like receptor (TLR) ligands. Macrophages derived from THP-1 cells transfected with pre-miRNA and anti-miRNA were stimulated with TLR ligands for 24 hours. We collected the supernatants for the quantification of inflammatory cytokine production. To identify the target genes, we overexpressed its miRNA and performed Agilent expression microarray. Transfection with reporter construct and pre-miRNA and anti-miRNA was performed to confirm suppression of target mRNA.

Results: We found that miR-204-3p was greatly decreased in the serum from FMF patients in attack. In vitro study, the expression of miR-204-3p was suppressed by LPS stimulation in macrophages derived from THP-1 cells. Inhibition of miR204-3p significantly induced the production of TLR4-related cytokines whereas overexpression of miR-204-3p inhibited their production. Bioinformatic analysis showed that miR-204-3p is predicted to target genes implicated in TLR pathway through regulation of PIK3 signaling. Reporter assay revealed that miR-204-3p directly suppressed the luciferase activity of 3'UTR of PIK3CG reporter construct.

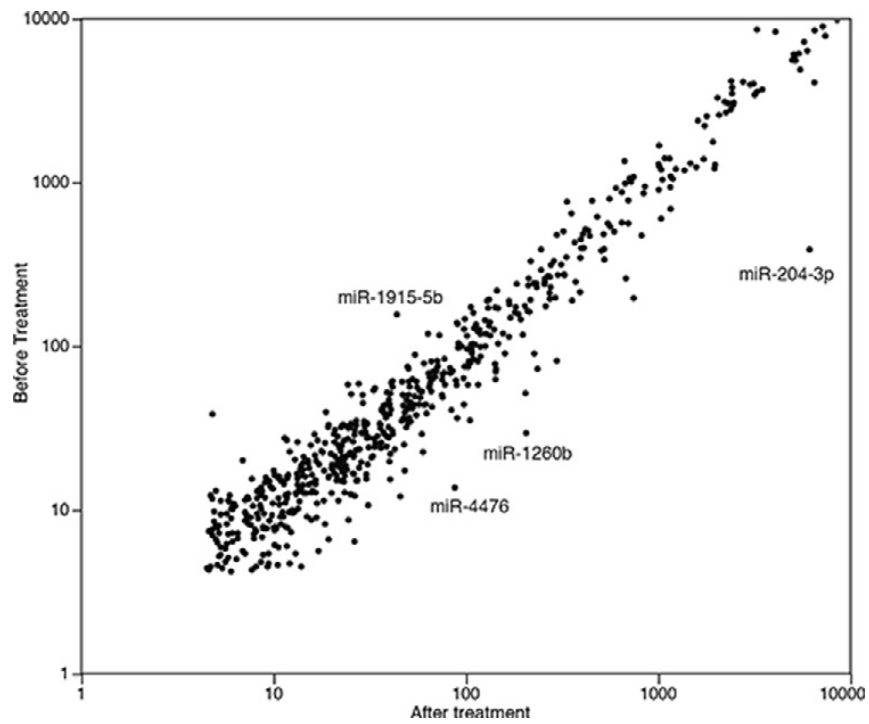

Conclusions: These data suggest that serum miR-204-3p has a potential as a useful biomarker among patients with FMF and that miR-204-3p plays a critical role as a suppressor to regulate the production of TLR4 related cytokines by targeting PIK3 signaling pathway.

Disclosure of Interest: None declared

DOI: 10.1136/annrheumdis-2017-eular.2813

\section{FRI0621 PREVALENCE OF DIFFERENT ORBITAL ANATOMIC STRUCTURES AFFECTION IN IGG4-RELATED OPHTHALMIC DISEASE: SINGLE CENTER EXPERIENCE}

V. Vasilyev $^{1}$, E. Sokol $^{1}$, T. Safonova ${ }^{2}$, S. Palshina ${ }^{1}$, N. Kokosadze ${ }^{3}$, A. Kovrigina ${ }^{4} .{ }^{1}$ Nasonova Research Institute of Rheumatology, Moscow, Russia ${ }^{2}$ Research Institute of Ophthalmic diseases; ${ }^{3}$ N.N. Blochin Russian Cancer Research Center; ${ }^{4}$ Hematology Research Center, Moscow, Russian Federation

Background: According to 2009 Japanese nationwide survey of IgG4-related disease (IgG4-RD), orbit is the leading site of affection [1]. However diagnostic criteria and nomenclature of IgG4-RD were developed a few years later [2,3] Objectives: To evaluate the peculiarities of clinical, laboratory, histological and immunohistochemical presentation of IgG4-related ophthalmic disease (IgG4ROD).

Methods: During 2004 - 2016, 82 patients were diagnosed with IgG4-RD 53 of whom had IgG4-ROD (men - 17, women - 36). The diagnoses of IgG4-RD and IgG4-ROD were established using comprehensive diagnostic criteria [2,3]. In all patients full clinical, ophtalmological, dental and serological (rheumatoid factor, C-reactive protein, IgG, IgG4, IgM, IgA, ANA, anti-Ro/La, C3/C4 complement) examination was carried. In all cases diagnosis was verified pathomorphologicaly with immunohystochemical staining (anti-CD 138, CD 68, IgG, IgG4, к-chain, $\lambda$-chain), but only in 43 patients the diagnosis of IgG4-RD was established on orbital tissues biopsy. Some patients at baseline were tested on B-cell clonality 